\title{
Persistence of SARS-CoV-2 specific B- and T-cell responses in convalescent COVID-19 patients 6-8 months after the infection
}

Natalia Sherina, Ph.D..$^{1^{*}}$, Antonio Piralla, M.D..$^{2^{*}}$, Likun Du, Ph.D. ${ }^{1^{*}}$, Hui Wan, M.S..$^{1^{*}}$, Makiko KumagaiBraesh, Ph.D. ${ }^{3}$, Juni Andréll, Ph.D. ${ }^{4}$, Sten Braesch-Andersen, Ph.D. ${ }^{5}$, Irene Cassaniti, Ph.D. ${ }^{2}$, Elena Percivalle, Ph.D. ${ }^{2}$, Antonella Sarasini, Ph.D ${ }^{2}$, Federica Bergami, B.S. ${ }^{2}$, Raffaella Di Martino, B.S. ${ }^{2}$, Marta Colaneri, M.D. ${ }^{6}$, Marco Vecchia, M.D. ${ }^{6}$, Margherita Sambo, M.D. ${ }^{6}$, Valentina Zuccaro, M.D ${ }^{6}$, Raffaele Bruno, M.D. ${ }^{6}$, Tiberio Oggionni, M.D. ${ }^{7}$, Federica Meloni, M.D. ${ }^{8}$, Hassan Abolhassani, Ph.D. ${ }^{9}$, Federico Bertoglio, Ph.D. ${ }^{10}$, Maren Schubert, Ph.D. ${ }^{10}$, Miranda Byrne-Steele, Ph.D. ${ }^{11}$, Jian Han, Ph.D. ${ }^{11}$, Michael Hust, Ph.D. ${ }^{10}$, Yintong Xue, Ph.D. ${ }^{12}$, Lennart Hammarström, Ph.D. ${ }^{9}$, Fausto Baldanti, Ph.D. ${ }^{2}{ }^{13 \#}$, Harold Marcotte, Ph.D. ${ }^{9 \#, ~ Q i a n g ~ P a n-H a m m a r s t r o ̈ m, ~ P h . D . ~}{ }^{1 \#}$

${ }^{1}$ Department of Biosciences and Nutrition, Karolinska Institutet, Huddinge, Sweden

${ }^{2}$ Molecular Virology Unit, Microbiology and Virology Department, Fondazione IRCCS Policlinico San Matteo, Pavia, Italy

${ }^{3}$ Division of Transplantation Surgery, CLINTEC, Karolinska Institutet at Karolinska University Hospital, Stockholm, Sweden

${ }^{4}$ Science for Life Laboratory, Department of Biochemistry and Biophysics, Stockholm University

${ }^{5}$ Mabtech AB Research Laboratory, Stockholm, Sweden

${ }^{6}$ Division of Infectious Diseases I, Fondazione IRCCS Policlinico San Matteo, Italy

${ }^{7}$ Unit of Respiratory Diseases, Department of Medical Sciences and Infective Diseases IRCCS Policlinico San Matteo Foundation, Pavia, Italy

${ }^{8}$ Section of Pneumology, Department of Internal Medicine, University of Pavia, Pavia, Italy

${ }^{9}$ Division of Clinical Immunology and Transfusion Medicine, Department of Laboratory Medicine, Karolinska Institutet at Karolinska University Hospital, Stockholm, Sweden

${ }^{10}$ Institute of Biochemistry, Biotechnology and Bioinformatics; Department of Biotechnology,

Technische Universität Braunschweig, Braunschweig, Germany

${ }^{11}$ HudsonAlpha Institute for Biotechnology, Huntsville, AL, USA

${ }^{12}$ Department of Immunology, Peking University Health Science Center, Beijing, China

${ }^{13}$ Department of Clinical, Surgical, Diagnostic and Paediatric Sciences, University of Pavia, Pavia, Italy

"These authors contributed equally to this work; "Co-senior authors.

Correspondence to: Dr. Qiang Pan-Hammarström, Department of Biosciences and Nutrition, Neo, Karolinska Institutet, SE14183 Huddinge, Sweden. qiang.pan-hammarstrom@ki.se 


\section{Summary}

Background The longevity of the immune response against SARS-CoV-2 is currently debated. We thus profiled the serum anti-SARS-CoV-2 antibody levels and virus specific memory B- and T-cell responses over time in convalescent COVID-19 patients.

Methods A cohort of COVID-19 patients from the Lombardy region in Italy who experienced mild to critical disease and Swedish volunteers with mild symptoms, were tested for the presence of elevated anti-spike and anti-receptor binding domain antibody levels over a period of eight months. In addition, specific memory B- and T-cell responses were tested in selected patient samples.

Results Anti-SARS-CoV-2 antibodies were present in $85 \%$ samples collected within 4 weeks after onset of symptoms in COVID-19 patients. Levels of specific IgM or IgA antibodies declined after 1 month while levels of specific IgG antibodies remained stable up to 6 months after diagnosis. Anti-SARS-CoV2 IgG antibodies were still present, though at a significantly lower level, in $80 \%$ samples collected at 6 8 months after symptom onset. SARS-CoV-2-specific memory B- and T-cell responses were developed in vast majority of the patients tested, regardless of disease severity, and remained detectable up to 6-8 months after infection.

Conclusions Although the serum levels of anti-SARS-CoV-2 IgG antibodies started to decline, virusspecific T and/or memory B cell responses increased with time and maintained during the study period (6-8 months after infection).

Funding European Union's Horizon 2020 research and innovation programme (ATAC), the Italian Ministry of Health, CIMED, the Swedish Research Council and the China Scholarship Council. 


\section{Introduction}

The emergence and spread of a novel coronavirus, SARS-CoV-2, has led to a pandemic with a major impact on global health. Currently, the immunological features associated with severity of the disease or protection remains largely unknown and whether the antibody titer is a marker for protective immunity against SARS-CoV-2 is currently debated. A robust adaptive immune response with presence of spike-specific neutralizing antibodies, memory B cells and circulating follicular helper T cells have been found in patients who have recovered from infection ${ }^{123}$. It is however still unclear how long the adaptive immunity to SARS-CoV-2 lasts after the natural infection. A relationship between a humoral immune response to SARS-CoV-2 infection and protection against reinfection has been shown in rhesus macaques ${ }^{4}$ but remains to be determined in humans. While a recent study in Iceland showed that the antibody response was maintained in $90 \%$ of convalescent patients for more than four months after onset of disease ${ }^{5}$, other studies suggest a rapid decay of anti-SARS-CoV-2 IgG in individuals with mild illness ${ }^{6}{ }^{7}$. Nevertheless, long-lived memory $T$ and $B$ cells could potentially be present and reactivated following a second exposure, thus providing immune protection.

The SARS-CoV-2 virus genome encodes four major structural proteins including the spike protein $(S)$, nucleoprotein $(N)$, membrane protein $(M)$, envelop $(E)$ and other membrane proteins (ORF3a, ORF7a) ${ }^{8}$. The primary target for an antibody-mediated response on the surface of SARS-CoV-2 virions is the homotrimeric S protein ${ }^{9}$. Antibodies targeting the $\mathrm{S}$ protein and its receptor-binding domain (RBD) are therefore of particular interest to combat the infection and a large range of RBD-specific monoclonal antibodies isolated from convalescent patients neutralize the virus both in vitro and in animal models 21011 .

Studies on the longevity of the adaptive immune response in convalescent COVID-19 patients may facilitate understanding of how immune protection develops and persists during the natural course of SARS-CoV-2 infection and provide useful information for the development of vaccines against this 
newly emerging virus. In this study, we aimed to assess the dynamics and longevity of the SARS-CoV2-specific immune responses in COVID-19 patients with broad spectrum of disease scores. Levels and Ig class of SARS-CoV-2-specific antibodies and development of memory B and T cells were evaluated in samples collected from 88 patients at different time points during a period of 8 months following initial symptoms.

\section{Methods}

\section{Patients and sample collection}

Screening of COVID-19 patient donors and sample collection were conducted at the Fondazione IRCCS Policlinico San Matteo in Pavia, Italy, a designated medical institution for COVID-19. Study inclusion criteria included subjects over 18 years of age, who were willing and able to provide informed consent, confirmed positivity of SARS-CoV-2 by real-time RT-PCR targeting the $E$ and $R d R p$ genes according to Corman et al. protocols ${ }^{12}$ and monitored until two subsequent samples with negative results. Between February 28 and October 10, 2020, 78 COVID-19 patients were recruited. Forty-seven donors had blood drawn at one single time point ranging from 7 to 240 days after symptom onset while 28 and 3 donors had blood taken at two or three time points, respectively. Disease severity was defined as mild (non-hospitalized), moderate (hospitalized, with lower respiratory tract infection, with dyspnea or not, but without oxygen support), severe (infectious disease/sub intensive ward with a need for oxygen and/or positive chest computed tomography scan, severe lower tract infections, with any oxygen support) and critical (intensive care unit (ICU) patients, intubated or with extracorporeal membrane oxygenation procedures).

The demographic and clinical characteristics of the patients are summarized in Table 1 and detailed in

S1. The patients had a median age of 63 years (range 32-89) with 45 (58\%) males and 33 (42\%) females. The degree of clinical severity of COVID-19 in cohort was mild $(n=5)$, moderate $(n=16)$, severe $(n=52)$ 
and critical ( $n=5)$. The most common underlying diseases were hypertension $40 / 78,51 \%)$, diabetes $(16 / 78,21 \%)$, heart disease $(12 / 78,15 \%)$ and obesity $(10 / 78,13 \%)$. The study was performed under the approval of the Institutional Review Board of Policlinico San Matteo (protocol number P_20200029440).

Twelve samples from 10 volunteers from Sweden (median age of 54 years, range 29-75) who had tested PCR- or serology-positive for SARS-CoV-2 and experienced mild symptoms were also included (Table 1 and S1). Blood samples were collected at 60-238 days after onset of symptoms. The study was approved by the ethics committee in Stockholm.

In addition, serum samples from 108 individuals (16 to 80 years of age), collected before the SARSCoV-2 pandemic (1995 to 2005) were used as historical negative controls for the ELISA and PBMCs from four healthy controls (median age 41 years, range 39-50) and seven additional buffy coats collected in Sweden before the SARS-CoV-2 pandemic (2011- January 2020) were included as negative controls for the B- and T-cell assays. Patients and samples tested in different assays were summarized in a flow chart (Figure 1).

\section{Detection of antibodies specific to SARS-CoV-2}

RBD-His protein was expressed in Expi293 cells and purified on Ni-NTA resin (\#88221, Thermofisher) followed by size-exclusion chromatography on a Superdex 200 gel filtration column in PBS ${ }^{13}$. S1-S2His (referred as S) protein was expressed baculovirus-free in High Five insect cells ${ }^{14}$ and purified on HisTrap excel column (Cytiva) followed by preparative size exclusion chromatography on 16/600 Superdex 200 kDa pg column (Cytiva) ${ }^{15}$.

High-binding Corning Half area plates (Corning \#3690) were coated over night at $4^{\circ} \mathrm{C}$ with S or RBD protein $(1.7 \mu \mathrm{g} / \mathrm{ml}$ for IgG and IgM; $2.0 \mu \mathrm{g} / \mathrm{ml}$ for $\lg A)$ in PBS; washed three times in PBS-Tween $(0.05 \%)$ 
and blocked with $2 \%$ BSA in PBS for $1 \mathrm{~h}$ at room temperature. Serum or plasma diluted 1:6400 (S IgG), 1:3200 (S IgM), 1:1600 (S IgA; RBD IgG, IgA, IgM) in 0.1\% BSA in PBS, was incubated for $1.5 \mathrm{~h}$ at room temperature. Plates were then washed and incubated for $1 \mathrm{~h}$ at room temperature with horseradish peroxidase (HRP)-conjugated goat anti-human IgG (Invitrogen \#A18805), goat anti-human IgM (Invitrogen \#A18835), or goat anti-human IgA (Jackson \#109-036-011) (all diluted 1:15 000 in 0.1\% BSAPBS). Bound antibodies were detected using tetramethylbenzidine substrate (Sigma \#T0440). The color reaction was stopped with $0.5 \mathrm{M} \mathrm{H}_{2} \mathrm{SO}_{4}$. Absorbance was measured at $450 \mathrm{~nm}$. Antibody levels were presented as arbitrary units $(\mathrm{AU} / \mathrm{ml})$, based on a standard curve made from a serially diluted highly positive serum pool.-A cut-off value for antibody positivity was defined for each antigen and isotype using receiver operating characteristic (ROC) curves, based on the antibody responses in historical controls ( $n=108)$ and COVID-19 patients (55 samples collected within 7-28 days after symptom onset). The cut-off value for positivity was set at $>0.03 \mathrm{AU} / \mathrm{ml}$ for anti-S IgG, $>0.5 \mathrm{AU} / \mathrm{ml}$ for anti-S IgA, $>2.5$ $\mathrm{AU} / \mathrm{ml}$ for anti-S IgM, $>14.8 \mathrm{AU} / \mathrm{ml}$ for anti-RBD IgG, $>0.08 \mathrm{AU} / \mathrm{ml}$ for anti-RBD IgA, and $>8.4 \mathrm{AU} / \mathrm{ml}$ for anti-RBD IgM, giving a specificity of $97 \%$ for $\operatorname{IgG}, 99 \%$ for $\lg A$ and $96 \% \operatorname{IgM}$. A previously described microneutralization assay ${ }^{16}{ }^{17}$ was used to determine the titers of SARS-CoV-2 neutralizing antibodies (NT-abs) in 37 samples. The neutralizing titer was the maximum dilution giving a reduction of $90 \%$ of the cytopathic effect.

\section{Isolation of PBMCs and RNA}

PBMCs were isolated from blood or buffy coat samples by standard density gradient centrifugation using Lymphoprep (Axis-Shield) and were cryopreserved and stored in liquid nitrogen until analysis. Total RNA was extracted from PBMCs by using RNeasy mini kit according to the manufacturer's protocol (Qiagen). 


\section{Enumeration of B cells secreting IgG antibodies specific for SARS-CoV-2 RBD and T cells secreting IFN- $\gamma$ and IL-2 in response to SARS-CoV-2 peptides}

PBMCs were incubated for four days in RPMI-1640 medium with $10 \% \mathrm{FCS}$, supplemented with the TLR7 and TLR8 agonist imidazoquinoline resiquimod (R848, $1 \mu \mathrm{g} / \mathrm{ml}$; Mabtech AB, Nacka, Sweden), and recombinant human IL-2 $(10 \mathrm{ng} / \mathrm{ml})$ for stimulation of memory B cells ${ }^{18}$. The ELISpot plates pre-coated with capturing monoclonal anti-human IgG antibodies were incubated with a total of 300000 or 30 000 pre-stimulated cells per well for detection of RBD-specific IgG and total IgG secreting cells, respectively. The number of B cells secreting IgG antibodies specific for SARS-CoV-2 RBD and cells secreting IgG (total IgG) were measured using the Human IgG SARS-CoV-2 RBD ELISpot ${ }^{\text {PLUS }}$ (ALP) kit according to the manufacturer's protocol (Mabtech $A B$ ).

IFN- $\gamma$ and IL-2 secreting T cells were detected using Human IFN- $\gamma /$ IL-2 SARS-CoV-2 FluoroSpot ${ }^{\text {PLus }}$ kits according to the manufacture's protocol (Mabtech AB). The plates pre-coated with capturing monoclonal anti-IFN- $\gamma$ and anti-IL-2 were incubated overnight in RPMI-1640 medium containing $10 \%$ FCS supplemented with a mixture containing the SARS-CoV-2 peptide pool (scanning or defined pools), anti-CD28 $(100 \mathrm{ng} / \mathrm{ml})$ and 300000 cells per well in a humidified incubators $\left(5 \% \mathrm{CO}_{2}, 37^{\circ} \mathrm{C}\right)$.

The SARS-CoV-2 S1 scanning pool contains 166 peptides from the human SARS-CoV-2 virus (\#3629-1, Mabtech AB). The peptides are 15-mers overlapping with 11 amino acids, covering the S1 domain of the S protein (amino acid 13-685). The SARS-CoV-2 S N M O defined peptide pool contains 47 synthetic peptides binding to human HLA, derived from the S, N, M ORF3a and ORF7a proteins (\#3622-1, Mabtech AB) ${ }^{19}$. The SARS-CoV-2 S2 $\mathrm{N}$ defined peptide pool contains 41 synthetic peptides binding to human HLA derived from the $\mathrm{S}$ and $\mathrm{N}$ proteins of the SARS-CoV-2 virus (\#3620-1, Mabtech AB) ${ }^{20}$. Results of ELISpot and Fluorospot assays were evaluated using an IRIS-reader and analyzed by IRIS software version 1.1.9 (Mabtech AB). The results were expressed as the number of spots per 300000 seeded cells after subtracting the background spots of the negative 
control. The cut-off value was set at the highest number of specific B- and T-cell spots from the negative controls.

\section{Statistical analysis}

Mann-Whitney $\mathrm{U}$ test was used for comparisons between groups in anti-SARS-CoV-2 antibody levels and numbers of specific memory B- and T-cells. Correlation analysis was performed using Spearman's rank correlation. A Wilcoxon signed-rank test was used for comparison paired samples. All analyses and data plotting were performed using GraphPad or R version 3.6.1.

\section{Role of the funding source}

The funders of the study had no role in study design, data collection, data analysis, data interpretation, or writing of the manuscript. The corresponding author had full access to all of the data in the study and had final responsibility for the decision to submit for publication.

\section{Results}

\section{The dynamics of the anti-SARS-CoV-2 antibody response in COVID-19 patients}

In order to evaluate the antibody response to SARS-CoV-2, 119 serum or plasma samples from 88 COVID-19 patients (78 from Italy and 10 from Sweden; Figure 1, Table 1 and Table S1) were tested by an in-house enzyme-linked immunosorbent assay (ELISA) for the presence of anti-S and anti-RBD antibodies. First, we examined the SARS-CoV-2-specific IgG, IgA, and IgM antibodies in 55 samples from COVID-19 patients collected during early phases of recovery (between 7-28 days after onset of disease symptoms) and 108 historical controls (samples collected prior to the SARS-CoV-2 pandemic). Significantly higher levels of anti-S and anti-RBD IgG, IgA and IgM antibodies ( $p<0.0001$ for all groups) were detected in patients as compared to historical controls (Fig. 2A-C). Anti-S IgG, IgA and IgM levels 
were increased in $85 \%, 78 \%$ and $76 \%$ of patients, respectively. A similar proportion of patients had elevated anti-RBD IgG, IgA and IgM levels with $85 \%, 62 \%$ and $67 \%$ being positive, respectively. Titers of anti-S and anti-RBD antibodies were highly correlated for all isotypes ( $r=0.95$ for $\lg G, r=0.77$ for $\lg A$, $\mathrm{r}=0.88$ for IgM) (Fig. 2D-F). SARS-CoV-2 NT-ab titers, measured by microneutralization test, correlated with levels of anti-S IgG ( $r=0.44)$, anti-S $\operatorname{lgA}(r=0.34)$ and anti-S IgM $(r=0.53)$, as well as with levels of anti-RBD IgG ( $r=0.39)$ and anti-RBD IgM ( $r=0.40)$ (data not shown).

Based on the symptoms presented at the time of COVID-19 diagnosis, patients were given a disease score ranging from mild, moderate, severe to critical (see MM). Analysis of anti-SARS-CoV-2 antibody levels did not show statistically significant difference in patients presented with severe and critical disease scores compared to mild or moderate disease groups ( $p=0.1444$ and $p=0.2943$ for anti-S IgG, $p=0.1203$ and $p=0.4672$ for anti-RBD IgG, respectively) (Fig. S1A-B). Notably, in six patients (11\%), rather low levels or even an absence of anti-S and anti-RBD IgG, IgA and IgM antibodies were observed. Sera from these individuals were obtained at median day 10.5 after symptom onset (range 7-22). A second sample taken at later time point was available for two of these patients ( 86 and 226 days after symptom onset), where both had become anti-S and anti-RBD IgG positive. For the other four samples (7\%), no second sample was available for analysis. These four individuals had a higher median age (79 years) compared to the median age for the entire patient cohort (62 years), had severe disease scores, and two of them later died from COVID-19 complications. When patients were divided based on sex, no statistically significant differences were observed in anti-S and anti-RBD antibody levels for all isotypes, except for anti-RBD IgG, where significantly higher levels were present in males compared to females with severe/critical disease scores $(p=0.0306)$ (Fig. S1C-D).

To examine the longevity of the anti-SARS-CoV-2 antibody response, we subsequently analyzed all 119 samples from 88 patients collected at different time points ( 7 to 240 days after symptom onset). AntiS and anti-RBD antibody levels were significantly increased already 7 to 14 days after symptom onset 
( $p<0.0001$ for all isotypes) and reached maximum at day 15 to 28 (Fig. 3) (days 7-14 to days 15-28, $p=0.0159$ and $p=0.0049$ for $\lg G, p=0.0080$ and $p=0.0029$ for $\lg A, p=0.0087$ and $p=0.0309$ for $\lg M$, respectively). After day 28, a significant decrease in anti-S and anti-RBD IgA and IgM antibody levels was observed (days $15-28$ to days $29-90, p=0.0184$ for anti-RBD IgA; days $15-28$ to days $91-180$, $\mathrm{p}<0.0001$ for anti-S and anti-RBD IgA and IgM; days 15-28 to days 181-240, $\mathrm{p}<0.0001$ for anti-S and anti-RBD IgA and IgM). No significant decrease in anti-S and anti-RBD IgG levels was present by days 91-180 (days $15-28$ to days $91-180, p=0.1847$ and $p=0.0544$, respectively), however a significant decline was observed by days $181-240$ (days $15-28$ to days $181-240, p=0.0003$ and $p=0.0002$, respectively). Importantly, a prominent anti-SARS-CoV-2 IgG response was still present in $80 \%(12 / 15)$ of the patients who were followed 181-240 days after onset of symptoms. These patients had mild $(n=5)$, moderate $(n=2)$ and severe $(n=5)$ disease scores at time of the diagnosis.

To further evaluate the dynamics of the anti-SARS-CoV-2 antibody response, we compared the antibody levels in paired samples from twenty-seven patients. The first sample was taken at median day 21 (range 7-64) after onset of symptoms and the paired second sample was taken at median day 126 (range 57-234). This analysis showed a significant decrease in anti-S IgA and IgM levels ( $p=0.0008$ and $p<0.0001$, respectively), and a significant decrease in anti-RBD IgA and IgM levels ( $p=0.0052$ and $p=0.0002$, respectively) with time (Fig. 4). No significant decline in anti-S and anti-RBD IgG levels was observed ( $p=0.1551)$. Taken together, these data suggest that an anti-SARS-CoV-2 antibody response was induced in a majority of COVID-19 patients in our study cohort and IgG antibodies remained present, although at lower levels, for at least 6-8 months after diagnosis.

\section{Induction of SARS-CoV-2 specific memory B and T cells}

To address the question whether SARS-CoV-2-specific memory B and T cells were formed and how long the B- and T- cell mediated responses persist in COVID-19 convalescent individuals, we analyzed 32 PBMC samples collected from 24 patients (mild=11, moderate $=4$, severe $=9$,Table S1). No or a 
negligible number of B cells secreting RBD-specific IgG antibodies were detected in samples from four healthy individuals and seven pre-pandemic buffy coats. Using the highest value observed from all negative controls as a cut-off, RBD-specific IgG producing B cells were detected in $33 \%(2 / 6)$ and $96 \%$ (25/26) of the patient samples collected 1-2 month and 3-8 months after onset of symptoms, respectively (Fig. 5A). Thus although the anti-RBD IgG levels declined over time (Fig. 5B), vast majority of the patients developed SARS-CoV-2-specific memory B cells, and these cells remained present in all patients followed up till latest date of study period ( $n=13,6-8$ months post symptom onset).

Furthermore, while no or negligible number of IL-2, IFN- $\gamma$ or IL-2/IFN- $\gamma$ producing T cells against three SARS-CoV-2 derived peptide pools were detected in the negative controls, such T cells were observed at a level above the cut-off in $17 \%$ to $100 \%$ of samples tested $1-2$ months after onset of symptoms, depending of the peptide pool tested (S1, S2 N or S N M O protein-derived) and in which cytokines were analyzed (Fig. 5C-E). At later time point (3-8 months after onset of symptoms), T cells specific for the SARS-CoV-2 S1 scanning pool and the defined peptide pool derived from the S, N, M, ORF-3a and ORF-7a proteins were found in $85 \%$ to $92 \%$ of patient samples, respectively, whereas T cells specific for the $\mathrm{S} 2 \mathrm{~N}$ defined peptide pool were observed in $54 \%$ to $77 \%$ of patient samples (Fig. 5C-E). Overall, T cell response against at least one of the SARS-CoV- 2 peptide pools was detectable in all patients $(n=6)$ tested at the early point (1-2 months), and such response maintained in vast majority $(96 \%, 22 / 23)$ of patients 3-8 months after onset of symptoms. Notably, the only patient who had no T cell response at 4 months, had a detectable memory B cell response. In two patients, a high number of T cells was also detected in the control test without adding the SARS-CoV-2 peptides, which may suggest an ongoing inflammation.

Moreover, for five paired patient samples collected at an early and later time points (median TP1 = 21 days, median TP2 $=204$ days $)$, a significant $(p<0.0001)$ increase in the number of virus-specific $B$ and T cells was observed in a second sample (Fig. 5F). Taken together, SARS-CoV-2-specific memory B and T 
cells were present in the vast majority of tested convalescent COVID-19 patients, regardless of the initial disease severity, suggesting that the adaptive immunity against SARS-CoV-2 during the natural course of infection is maintained at least for 6-8 months.

\section{Discussion}

In the current study, we measured anti-S and anti-RDB IgG, IgA and IgM antibody levels using normalization against a serially diluted highly positive reference serum pool and by setting a cut-off value based on historical control samples. Our data revealed that an anti-SARS-CoV-2 antibody response was present in a majority of COVID-19 patients as early as two weeks after onset of symptoms, and the level of anti-S and anti-RDB IgG remained stable up to 6 months after diagnosis followed by a decline at month $6-8$, while a decrease in anti-S and anti-RDB IgA and IgM levels was observed already between 1 and 3 months after onset of diseases. Our results are in line with previous studies showing a similar longevity and pattern of anti-SARS-CoV-2 antibody response with antibody levels reaching a peak at 23 days following symptom onset and being maintained for at least 4 months 521222324 , yet contradictory to others, where a low prevalence and rapid decay (within 3 months) of anti-SARS-CoV-2 antibodies in COVID-19 patients with mild or severe disease were observed ${ }^{625}$. In agreement with other reports, we also observed higher anti-RBD IgG antibody titers in men who were more severely affected by SARS-CoV-2 infection while seven percent of patients with a severe disease score in our study did not develop, or had extremely low level of antibodies, after being infected, suggesting that they mounted a weaker anti-viral immune response $5^{725}$. Higher levels of antibodies in individuals with severe diseases and in male patients might be due to a higher viral load, longer duration of viral shedding 2627 or other host/genetic factors 2829 .

Previously reported conflicting findings in prevalence and longevity of anti-SARS-CoV-2 antibody response may result from an absence of a standard assay to measure anti-SARS-CoV-2 antibodies, as a majority of reported studies are based on using different types of SARS-CoV-2 antigens (RBD, S or N 
proteins). The discrepant results between studies could also be explained by differences between COVID-19 cohorts, as the treatment used, age and sex of study subjects could affect the outcome ${ }^{30}$. Although the protective role of antibodies against SARS-CoV-2 remains unknown, levels of anti-RBD antibodies seemingly correspond to plasma neutralization activity ${ }^{31}{ }^{24}$. While our study cohort is relatively small, the inclusion of Italian and Swedish patients with a different spectrum of disease and the longest follow-up time reported so far (up to 6-8 months), can help to solve the current debate about the persistence of SARS-CoV-2 specific antibodies. It has been reported that antibodies against the two other coronavirus, SARS-CoV and MERS-CoV, could still be detected one to three years after infection onset ${ }^{26}$, suggesting that SARS-CoV-2 specific antibodies may be present for even longer period than we have observed thus far.

Studies reported until now have mainly been focused on the longevity of the specific antiviral antibody responses. However, the development of memory B and T cell is critical for long-term protection and the longitudinal dynamics of these memory cells remains poorly resolved. Our results show that the majority of patients, irrespective of disease severity, can mount specific memory B- and T-cell responses, which remain present at least 6-8 months post symptom onset. These findings are consistent with preprints showing, using flow cytometry, that RBD-specific memory B cells are generated and maintained up to 3-5 months post-SARS-CoV-2 infection in predominantly mildmoderate cohorts 3233 . Importantly, we show that these memory B cells are maintained and can secrete RBD-specific IgG antibodies following stimulation.

Previously, it has been shown that S1 and other SARS-CoV-2 proteins derived peptides induce specific T-cell responses in patients with mild to severe disease 1-3 months post symptoms ${ }^{13} 323536$. More recently, it was also reported that SARS-CoV-2-specific T cells are maintained at least 6 months following primary infection in all tested COVID-19 patients with mild to moderated disease ${ }^{37}$. Our results confirm and extend earlier findings, showing that SARS-CoV-2-specific, IL-2 and/or IFN- $\gamma$ 
producing cells are present in samples collected from patients with mild to severe diseases, up to 6-8 months post infection. Some studies also noted that $\mathrm{T}$ cell responses directed against the $\mathrm{S}$ and/or $\mathrm{M}$ of SARS-CoV-2 are present in $25-50 \%$ of unexposed healthy blood donors, consistent with a high degree of potentially cross-reactive T cell immunity in the general population ${ }^{136}{ }^{38}$. In our study, the significant increase in number of T cells reacting with SARS-CoV-2-derived peptides from consecutive samples collected at 2-3 weeks and 6-8 months from the same patients strongly suggests a specific response to the SARS-CoV-2 infection, although induction of cross-reactive memory $T$ cells resulting from priming by common cold coronaviruses (e.g. OC43, 229E, NL63 and HKU1) cannot be totally excluded ${ }^{39}$. The reason we could not detect significant number of cross reactive T cells against the virus might be due to small number of samples tested and/or longer stimulation with the peptides in some other studies ${ }^{39}$, however cross reactive T cell were also observed in studies using stimulation for a shorter time $(9$ to 24 hours ) $)^{13638}$.

The detection of memory S1-specific T cells marked by production of IL-2, IFN- $\gamma$ and dual production of those cytokine is indicative of induction of T cells with both effector and proliferative potential in vivo. IFN- $\gamma$ producing T cells is a hallmark of immunity against intracellular pathogens and although it was not tested in our study, SARS-CoV-2-specific IFN- $\gamma$ producing T cells were previously shown to be of $\mathrm{CD}^{+}$(Th1-like) or $\mathrm{CD}^{+}$cytotoxic phenotype ${ }^{136}$. It was shown that convalescent patient donors with undetectable antibodies against the S1 protein of SARS-CoV-2 had T-cell responses more strongly directed against the $\mathrm{M}$ than the $\mathrm{S} 1$ protein ${ }^{37}$. Furthermore, a Th1-biased cellular immune response of S-specific IFN- $\gamma$ positive $\mathrm{CD}^{+}{ }^{+}$T cells to pooled S peptides was detected in the majority of monkeys vaccinated with $\mathrm{S}$ protein and was associated with induction of specific and neutralizing anti-S antibodies ${ }^{40}$. Our results suggest that the use of $\mathrm{S}$ protein as an immunogen for vaccination has the potential to induce memory $\mathrm{T}$ and $\mathrm{B}$ cell specific for the $\mathrm{S}$ protein and RBD in humans. 
Importantly, the decrease in serum IgG antibody levels observed over time in our study was followed by a significant increase in the number of specific memory $B$ and $T$ cells, that could potentially contribute to protection from SARS-CoV-2 reinfection ${ }^{32}{ }^{34}$. However, the detection of antibodies to SARS-CoV-2, including neutralizing antibodies, as well as memory B cell and T cell over a long period does not necessarily indicate protective and long-term immunity, and a correlate of protection still needs to be established. Studies on common human coronaviruses show that neutralizing antibodies are induced and reinfections with all seasonal coronaviruses occur usually within three years ${ }^{26}$. A recent study showed that the duration of protective immunity against common cold coronavirus may last 6 to 12 months ${ }^{41}$ although others reported that repeat infections are generally associated with milder symptoms and a lower viral load 26 42. Single intravenous administration of neutralizing antibodies against the spike protein in outpatients with mild or moderate COVID-19 has been shown to reduce the viral load and reduced length of hospitalization ${ }^{43}$. Furthermore, infection with SARSCoV-2 and vaccine against the spike protein can protect rhesus macaque from a challenge infection ${ }^{4}$. It is thus likely that antibodies and cell-mediated immunity will decrease the risk of reinfection and attenuate the severity in case of reinfection. Characterization of immune responses prior to a known exposure or period of risk is required to identify a correlate of protection ${ }^{26}$. We thus plan to expand our cohort and follow it over longer intervals of time in order to evaluate the maintenance of immunological memory.

The presence of high level of SARS-CoV-2 specific memory B and T cells in the majority of patients, 68 months after infection, suggests that immunity after infection could be at least transiently protective and that development of long-term protective immunity through vaccination might be possible. The discovery of $T$ cell reactivity against $S$ protein epitopes and antibodies against the RBD domain suggests that vaccine development using the $S$ protein to induce antibodies that target RBD is a plausible approach ${ }^{35}$. To meet the urgent need for SARS-CoV-2 vaccine development, we propose that in addition to analysis of specific antibody responses and their longevity, the development of memory B 
and T cells, the main components of long-term immunity, as well as correlation with protection from reinfection should be considered.

\section{Contributors}

NS performed the ELISA experiment; A.P., I.C., E.P., A.S., F.Bergami, R.M., H.A., L.D., and F.Ba. contributed to patient data curation, sample preparation and neutralization assay; L.D., M.K.-B. and S.B.-A. designed and/or performed the ELISpot and FluoroSpot experiments; M.V., M.Sa., V.Z., R.B., T.O., and F.M. contributed to the enrollment of patients, patient management and collection of clinical data. J.A., F.Bertoglio, M.Sh. and M.H. performed the production and purification of proteins. N.S., L.D., H.W., M.K.B., M.B.S., H.J., H.A., Y..X, L.H., H.M., and Q.P.-H. contributed to the analysis and interpretation of data. N.S., H.M. and Q.P.-H. drafted the manuscript. H.M., F.B. and Q.P.-H. conceived and supervised the study.

\section{Declaration of interests}

S.B.-A. is a member of the advisory board of Mabtech AB. The other authors declare no competing interests.

\section{Acknowledgments}

We thank all patients, blood donors and clinicians for their contribution. This project was supported by the European Union's Horizon 2020 research and innovation programme (ATAC, No 101003650), the Italian Ministry of Health (Ricerca Finalizzata grant no. GR-2013-02358399), CIMED, the Swedish Research Council and funds from the Karolinska Institutet. H.W. was supported by a scholarship from the China Scholarship Council. 


\section{References}

1 Grifoni A, Weiskopf D, Ramirez SI, et al. Targets of T Cell Responses to SARS-CoV-2 Coronavirus in Humans with COVID-19 Disease and Unexposed Individuals. Cell 2020; 181: 1489-1501.e15.

2 Robbiani DF, Gaebler C, Muecksch F, et al. Convergent antibody responses to SARS-CoV-2 in convalescent individuals. Nature 2020; 584: 437-42.

3 Juno JA, Tan H-X, Lee WS, et al. Humoral and circulating follicular helper $\mathrm{T}$ cell responses in recovered patients with COVID-19. Nat Med 2020; 26: 1428-34.

4 Chandrashekar A, Liu J, Martinot AJ, et al. SARS-CoV-2 infection protects against rechallenge in rhesus macaques. Science 2020; 369: 812-7.

5 Gudbjartsson DF, Norddahl GL, Melsted P, et al. Humoral Immune Response to SARS-CoV-2 in Iceland. N Engl J Med 2020; (in press).

6 Ibarrondo FJ, Fulcher JA, Goodman-Meza D, et al. Rapid Decay of Anti-SARS-CoV-2 Antibodies in Persons with Mild Covid-19. N Engl J Med 2020; 383: 1085-7.

7 Long Q-X, Tang X-J, Shi Q-L, et al. Clinical and immunological assessment of asymptomatic SARSCoV-2 infections. Nat Med 2020; 26: 1200-4.

8 Naqvi AAT, Fatima K, Mohammad T, et al. Insights into SARS-CoV-2 genome, structure, evolution, pathogenesis and therapies: Structural genomics approach. Biochim Biophys Acta Mol Basis Dis 2020; 1866: 165878.

9 Zhang Y, Kutateladze TG. Molecular structure analyses suggest strategies to therapeutically target SARS-CoV-2. Nat Commun 2020; 11: 2920.

10 Hassan AO, Case JB, Winkler ES, et al. A SARS-CoV-2 Infection Model in Mice Demonstrates Protection by Neutralizing Antibodies. Cell 2020; 182: 744-753.e4.

11 Rogers TF, Zhao F, Huang D, et al. Isolation of potent SARS-CoV-2 neutralizing antibodies and protection from disease in a small animal model. Science 2020; 369: 956-63.

12 Corman VM, Landt O, Kaiser M, et al. Detection of 2019 novel coronavirus (2019-nCoV) by real-time RT-PCR. Euro Surveill; 25: 2000045.

13 Amanat F, Stadlbauer D, Strohmeier $S$, et al. A serological assay to detect SARS-CoV-2 seroconversion in humans. Nat Med 2020; 26: 1033-6.

14 Bleckmann M, Schürig M, Endres $M$, et al. Identifying parameters to improve the reproducibility of transient gene expression in High Five cells. PLoS One 2019; 14: e0217878.

15 Bertoglio F, Meier D, Langreder N, et al. SARS-CoV-2 neutralizing human recombinant antibodies selected from pre-pandemic healthy donors binding at RBD-ACE2 interface. bioRxiv 2020; 2020.06.05.135921.

16 Percivalle E, Cambiè G, Cassaniti I, et al. Prevalence of SARS-CoV-2 specific neutralising antibodies in blood donors from the Lodi Red Zone in Lombardy, Italy, as at 06 April 2020. Euro Surveill 2020; 25: 2001031. 
17 Bonelli F, Sarasini A, Zierold C, et al. Clinical and Analytical Performance of an Automated Serological Test That Identifies S1/S2-Neutralizing IgG in COVID-19 Patients Semiquantitatively. J Clin Microbiol 2020; 58: e01224-20.

18 Jahnmatz M, Kesa G, Netterlid E, Buisman A-M, Thorstensson R, Ahlborg N. Optimization of a human IgG B-cell ELISpot assay for the analysis of vaccine-induced B-cell responses. J Immunol Methods 2013; 391: 50-9.

19 Peng Y, Mentzer AJ, Liu G, et al. Broad and strong memory CD4 + and CD8 + T cells induced by SARSCoV-2 in UK convalescent individuals following COVID-19. Nat Immunol 2020; 21: 1336-1346.

20 Ahmed SF, Quadeer AA, McKay MR. Preliminary Identification of Potential Vaccine Targets for the COVID-19 Coronavirus (SARS-CoV-2) Based on SARS-CoV Immunological Studies. Viruses 2020; 12: 254.

21 Bölke E, Matuschek C, Fischer JC. Loss of Anti-SARS-CoV-2 Antibodies in Mild Covid-19. N Engl J Med 2020; 383: 1694-1698.

22 Iyer AS, Jones FK, Nodoushani A, et al. Persistence and decay of human antibody responses to the receptor binding domain of SARS-CoV-2 spike protein in COVID-19 patients. Sci Immunol 2020; 5: eabe0367.

23 Isho B, Abe KT, Zuo M, et al. Persistence of serum and saliva antibody responses to SARS-CoV-2 spike antigens in COVID-19 patients. Sci Immunol 2020; 5: eabe5511.

24 Wajnberg A, Amanat F, Firpo A, et al. Robust neutralizing antibodies to SARS-CoV-2 infection persist for months. Science 2020; 5: eabe0367.

25 Kutsuna S, Asai U, Matsunaga A. Loss of Anti-SARS-CoV-2 Antibodies in Mild Covid-19. N Eng J Med 2020; 393: 1694-1698.

26 Huang AT, Garcia-Carreras B, Hitchings MDT, et al. A systematic review of antibody mediated immunity to coronaviruses: kinetics, correlates of protection, and association with severity. Nat Commun 2020; 11: 4704.

27 Zheng S, Fan J, Yu F, et al. Viral load dynamics and disease severity in patients infected with SARSCoV-2 in Zhejiang province, China, January-March 2020: retrospective cohort study. BMJ 2020; 369: m1443.

28 Bastard P, Rosen LB, Zhang Q, et al. Autoantibodies against type I IFNs in patients with lifethreatening COVID-19. Science 2020; 370: eabd4585.

29 Zhang Q, Bastard P, Liu Z, et al. Inborn errors of type I IFN immunity in patients with life-threatening COVID-19. Science 2020; 370; eabd4570.

30 Yang OO, Ibarrondo FJ. Loss of Anti-SARS-CoV-2 Antibodies in Mild Covid-19. N Eng J Med 2020; 393: 1694-8.

31 Premkumar L, Segovia-Chumbez B, Jadi R, et al. The receptor binding domain of the viral spike protein is an immunodominant and highly specific target of antibodies in SARS-CoV-2 patients. Sci Immunol 2020; 5: eabc8413. 
32 Rodda LB, Netland J, Shehata L, et al. Functional SARS-CoV-2-specific immune memory persists after mild COVID-19. medRxiv 2020; 2020.08.11.20171843.

33 Vaisman-Mentesh A, Dror $Y$, Tur-Kaspa R, et al. SARS-CoV-2 specific memory B cells frequency in recovered patient remains stable while antibodies decay over time. medRxiv 2020; 2020.08.23.20179796.

34 Wheatley AK, Juno JA, Wang JJ, et al. Evolution of immunity to SARS-CoV-2. medRxiv 2020; 2020.09.09.20191205.

35 Zhang F, Gan R, Zhen Z, et al. Adaptive immune responses to SARS-CoV-2 infection in severe versus mild individuals. Signal Transduct Target Ther 2020; 5: 156.

36 Sekine T, Perez-Potti A, Rivera-Ballesteros O, et al. Robust T Cell Immunity in Convalescent Individuals with Asymptomatic or Mild COVID-19. Cell 2020; 183: 158-68.

37 Zuo J, Dowell A, Pearce $\mathrm{H}$, et al. Robust SARS-CoV-2-specific T-cell immunity is maintained at 6 months following primary infection. bioRxiv 2020; 2020.11.01.362319.

38 Braun J, Loyal L, Frentsch M, et al. SARS-CoV-2-reactive T cells in healthy donors and patients with COVID-19. Nature 2020; (in press).

39 Nelde A, Bilich T, Heitmann JS, et al. SARS-CoV-2-derived peptides define heterologous and COVID19-induced T cell recognition. Nat Immunol 2020; (in press).

$40 \mathrm{Yu}$ J, Tostanoski LH, Peter L, et al. DNA vaccine protection against SARS-CoV- 2 in rhesus macaques. Science 2020; 369: 806-11.

41 Edridge AWD, Kaczorowska J, Hoste ACR, et al. Seasonal coronavirus protective immunity is shortlasting. Nat Med 2020; (in press).

42 Callow KA, Parry HF, Sergeant M, Tyrrell DA. The time course of the immune response to experimental coronavirus infection of man. Epidemiol Infect 1990; 105: 435-46.

43 Chen W, Zhang J, Qin X, et al. SARS-CoV-2 neutralizing antibody levels are correlated with severity of COVID-19 pneumonia. Biomed \& Pharmacother 2020; 130: 110629. 
Figure 1 Flow chart illustrating the study design.

Figure 2. Anti-SARS-CoV-2 antibody response in COVID-19 patients. Levels of anti-S and anti-RBD IgG, $\operatorname{lgA}$ and IgM antibodies in historical controls and COVID-19 patients (A, B, C). Correlation between titers of anti-S and anti-RBD IgG, $\lg A$, and $\operatorname{IgM}(\mathbf{D}, \mathbf{E}, \mathbf{F})$. Symbols represent individual subjects; horizontal black lines indicate the median. The dashed red line indicates the cut-off value for elevated anti-S and anti-RBD antibody levels (0.03 and $14.81 \mathrm{AU} / \mathrm{ml}$ for IgG, 0.5 and $0.08 \mathrm{AU} / \mathrm{ml}$ for $\operatorname{lgA}, 2.5$ and $8.4 \mathrm{AU} / \mathrm{ml}$ for IgM, respectively) defined using receiver operating characteristic (ROC) curves, based on the antibody responses in historical controls $(n=108)$ and COVID-19 patients $(n=55)$. Percentages in graphs A, B and C show the frequency of antibody positive individuals. Statistical significance determined by a non-parametric Mann-Whitney $U$ test $(A, B, C)$, correlation analysis was performed by Spearman's rank correlation $(\mathrm{D}, \mathrm{E}, \mathrm{F}) . * * * * \mathrm{p}<0.0001$

Figure 3. Longevity of the anti-SARS-CoV-2 antibody response in COVID-19 patients. Anti-S and antiRBD IgG, IgA and IgM antibody response in COVID-19 patients during the time following the diagnosis and recovery (A, B). In total, 119 samples were collected from 88 patients. Samples were taken at 5 study periods: $1-2$ weeks ( $n=19), 2-4$ weeks $(n=36), 1-3$ months $(n=22), 3-6$ months $(n-27)$, and 6-8 months ( $n=15)$ after symptom onset. Symbols represent individual subjects; horizontal black lines indicate the median. The dashed red line indicates the cut-off value for elevated anti-S and anti-RBD antibody levels (0.03 and $14.81 \mathrm{AU} / \mathrm{ml}$ for $\operatorname{lgG}, 0.5$ and $0.08 \mathrm{AU} / \mathrm{ml}$ for $\operatorname{lgA}, 2.5$ and $8.4 \mathrm{AU} / \mathrm{ml}$ for $\operatorname{lgM}$, respectively) defined using receiver operating characteristic (ROC) curves, based on the antibody responses in historical controls $(n=108)$ and COVID-19 patients $(n=55)$. Statistical significance determined by a non-parametric Mann-Whitney $U$ test. ${ }^{*} p \leq 0.05,{ }^{* *} p \leq 0.001,{ }^{* * *} p \leq 0.001$, $* * * \mathrm{p}<0.0001$ 
Figure 4. Dynamics of the anti-SARS-CoV-2 antibody levels in paired samples from COVID-19 patients. Levels of anti-S (A, B, C) and anti-RBD (D, E, F) IgG, IgA and IgM antibodies in twenty-seven pairs of COVID-19 patients measured at timepoint 1 (TP1, median day 21, range 7-64) and timepoint 2 (TP2, median day 126, range 57-234) and presented on group (left panel) or individual (right panel) level. Symbols represent individual subjects; horizontal black lines indicate the median. Statistical significance determined by a non-parametric Mann-Whitney U test. ${ }^{* *} p \leq 0.001,{ }^{* * *} p \leq 0.001$

Figure 5. SARS-CoV-2-specific $T$ and memory B cell responses in COVID19 patients. RBD-specific memory B cells from control and COVID-19 patient samples (A). Dynamic of RBD-specific memory B cell and serum anti-RBD IgG levels in COVID-19 patient samples over time (B). SARS-CoV-2-specific T cells specific for the S1, S2N and S N M O protein derived peptides pools and producing IL-2 (C), IFN- $\gamma$ (D), or IFN- $\gamma$ and IL-2 (E) in control and COVID-19 patient samples respectively. Increase in specific memory B cells and IFN- $\gamma / \mathrm{IL}-2$ producing T cells specific for the S1, S2N and S N M O protein derived peptides pools in five patients (with mild (CP45), moderate (CP08, CP09) or severe (CP06, CP34) disease) at time point 1 (TP1) and time point 2 (TP2) (F). The results were expressed as the number of spots per 300000 seeded cells after subtracting the background spots of the negative control. The red line indicates the median value of the group. The cut-off value was set at the highest number of specific $B$ and T cell spots for the negative controls. P value was calculated by Mann-Whitney $U$ test. ${ }^{* *} p \leq 0.01$, $* * * p \leq 0.001, * * * * p \leq 0.0001$ 
Table 1. Summary of demographic and clinical characteristics in COVID-19 positive individuals

\begin{tabular}{|c|c|c|c|c|}
\hline & \multicolumn{2}{|c|}{ Italian cohort } & \multicolumn{2}{|c|}{ Swedish cohort } \\
\hline \multicolumn{5}{|l|}{ Demographic } \\
\hline Number & 78 & & 10 & \\
\hline Age, median (range) & 63.0 & & 53.5 & \\
\hline Male & $58 \%$ & $(45 / 78)$ & $50 \%$ & $(5 / 10)$ \\
\hline Female & $42 \%$ & $(33 / 78)$ & $50 \%$ & $(5 / 10)$ \\
\hline \multicolumn{5}{|l|}{ Disease severity } \\
\hline Mild & $6 \%$ & $(5 / 78)$ & $100 \%$ & $(10 / 10)$ \\
\hline Moderate & $21 \%$ & $(16 / 78)$ & $0 \%$ & $(0 / 10)$ \\
\hline Severe & $67 \%$ & $(52 / 78)$ & $0 \%$ & $(0 / 10)$ \\
\hline Critical & $6 \%$ & $(5 / 78)$ & $0 \%$ & $(0 / 10)$ \\
\hline \multicolumn{5}{|l|}{ Symptoms } \\
\hline Fever & $96 \%$ & $(75 / 78)$ & $40 \%$ & $(4 / 10)$ \\
\hline Cough & $67 \%$ & $(52 / 78)$ & $40 \%$ & $(4 / 10)$ \\
\hline Dyspnea & $47 \%$ & $(37 / 78)$ & $0 \%$ & $(0 / 10)$ \\
\hline Asthenia & $12 \%$ & $(9 / 78)$ & $60 \%$ & $(6 / 10)$ \\
\hline Diarrhea & $9 \%$ & $(7 / 78)$ & $0 \%$ & $(0 / 10)$ \\
\hline Anosmia & $4 \%$ & $(3 / 78)$ & $50 \%$ & $(5 / 10)$ \\
\hline Hypoxia & $1 \%$ & $(1 / 78)$ & $0 \%$ & $(0 / 10)$ \\
\hline Myalgia & $1 \%$ & $(1 / 78)$ & $50 \%$ & $(5 / 10)$ \\
\hline \multicolumn{5}{|l|}{ Past medical history } \\
\hline Hypertension & $51 \%$ & $(40 / 78)$ & $0 \%$ & $(0 / 10)$ \\
\hline Diabetes & $21 \%$ & $(16 / 78)$ & $0 \%$ & $(0 / 10)$ \\
\hline Hearth diseases & $15 \%$ & $(12 / 78)$ & $0 \%$ & $(0 / 10)$ \\
\hline Obesity & $13 \%$ & $(10 / 78)$ & $0 \%$ & $(0 / 10)$ \\
\hline $\mathrm{HCV}$ & $10 \%$ & $(8 / 78)$ & $0 \%$ & $(0 / 10)$ \\
\hline Lung diseases & $5 \%$ & $(4 / 78)$ & $0 \%$ & $(0 / 10)$ \\
\hline Tumor & $5 \%$ & $(4 / 78)$ & $0 \%$ & $(0 / 10)$ \\
\hline Other comorbidities & $41 \%$ & $(32 / 78)$ & $10 \%$ & $(1 / 10)$ \\
\hline More than one comorbidity & $49 \%$ & $(38 / 78)$ & $0 \%$ & $(0 / 10)$ \\
\hline $\begin{array}{l}\text { More than two } \\
\text { comorbidities }\end{array}$ & $29 \%$ & $(23 / 78)$ & $0 \%$ & $(0 / 10)$ \\
\hline \multicolumn{5}{|l|}{ Severity } \\
\hline Oxygen therapy & $73 \%$ & $(57 / 78)$ & $0 \%$ & $(0 / 10)$ \\
\hline Intensive care unit (ICU) & $6 \%$ & $(5 / 78)$ & $0 \%$ & $(0 / 10)$ \\
\hline
\end{tabular}




\section{Figure 1}

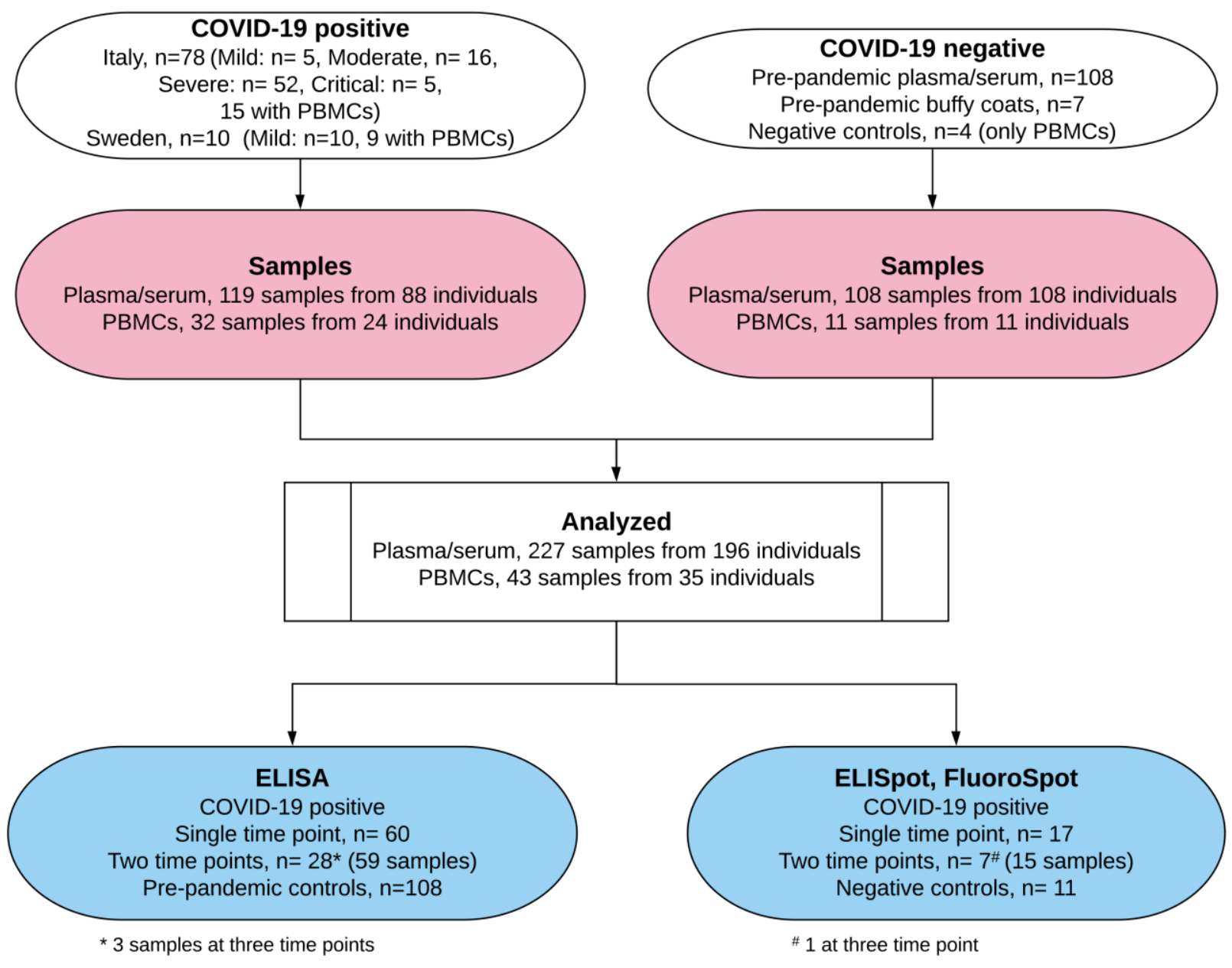




\section{Figure 2}

A

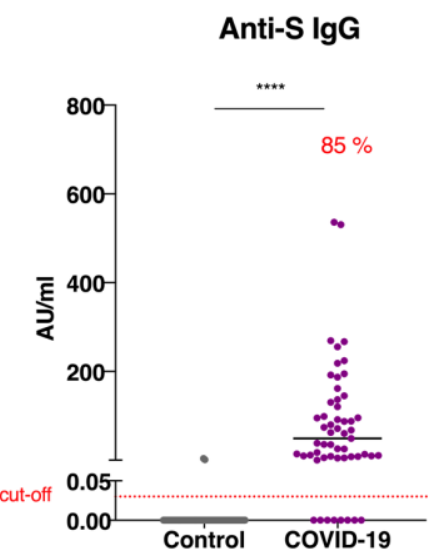

B

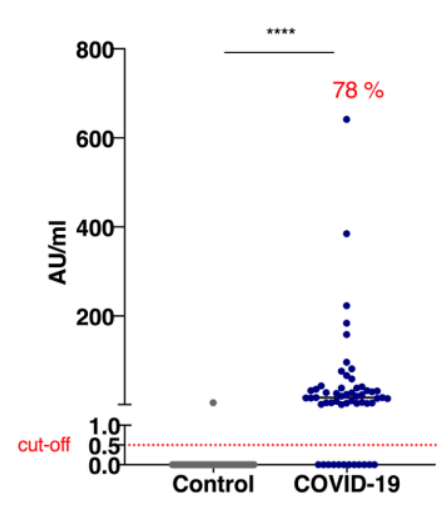

C

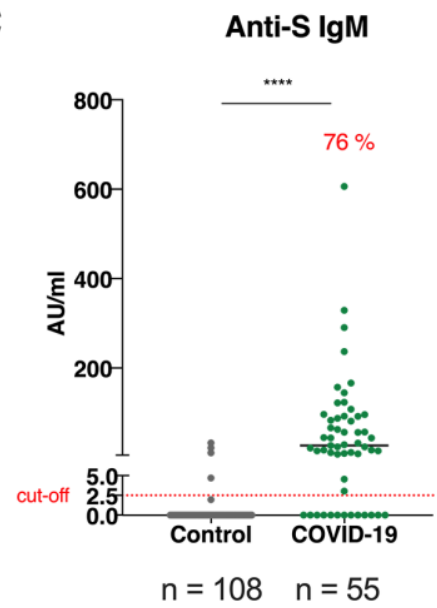

Anti-RBD IgG

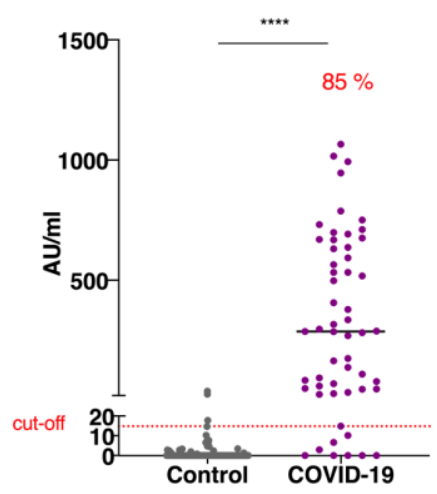

Anti-RBD IgA

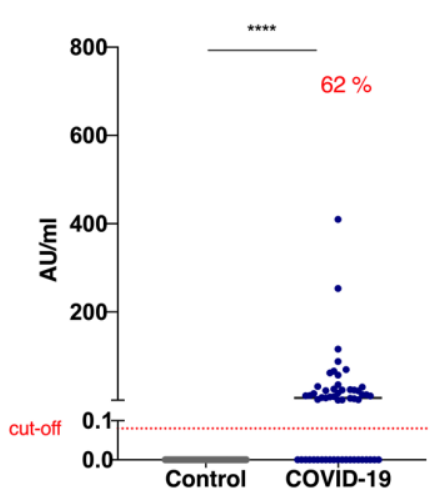

Anti-RBD IgM

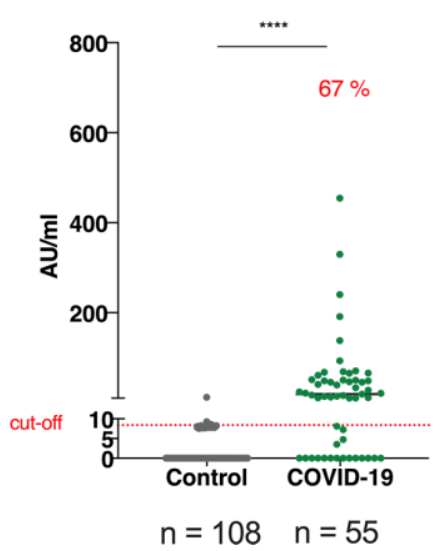

D

Spike-RBD IgG correlation

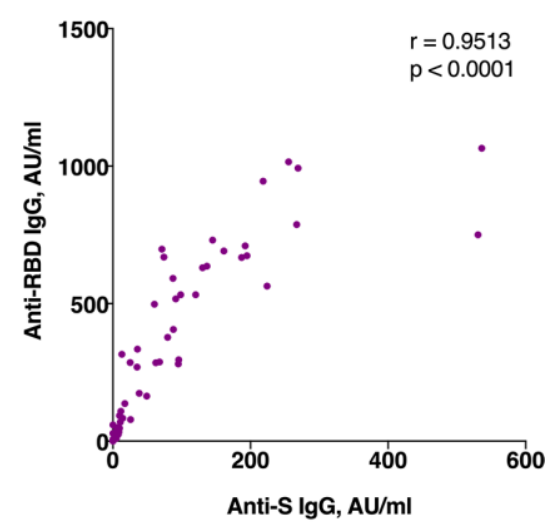

E
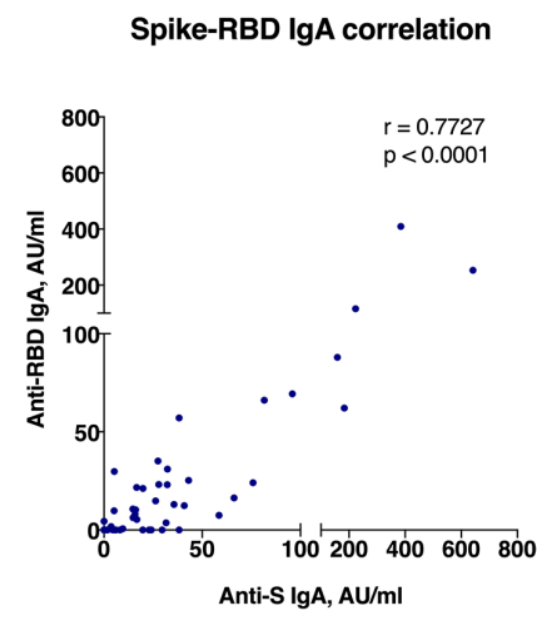

F

Spike-RBD IgM correlation

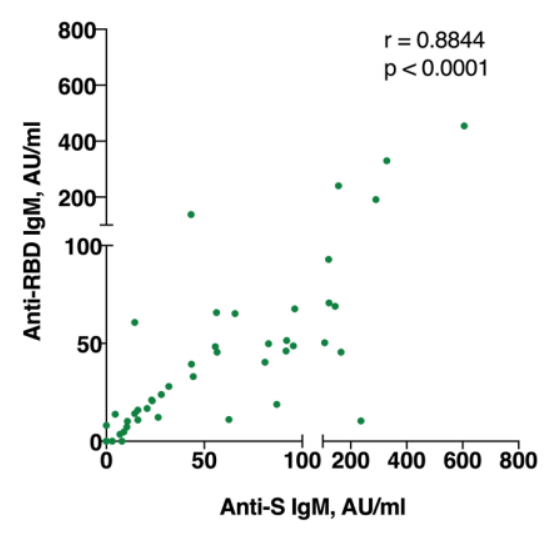




\section{Figure 3}
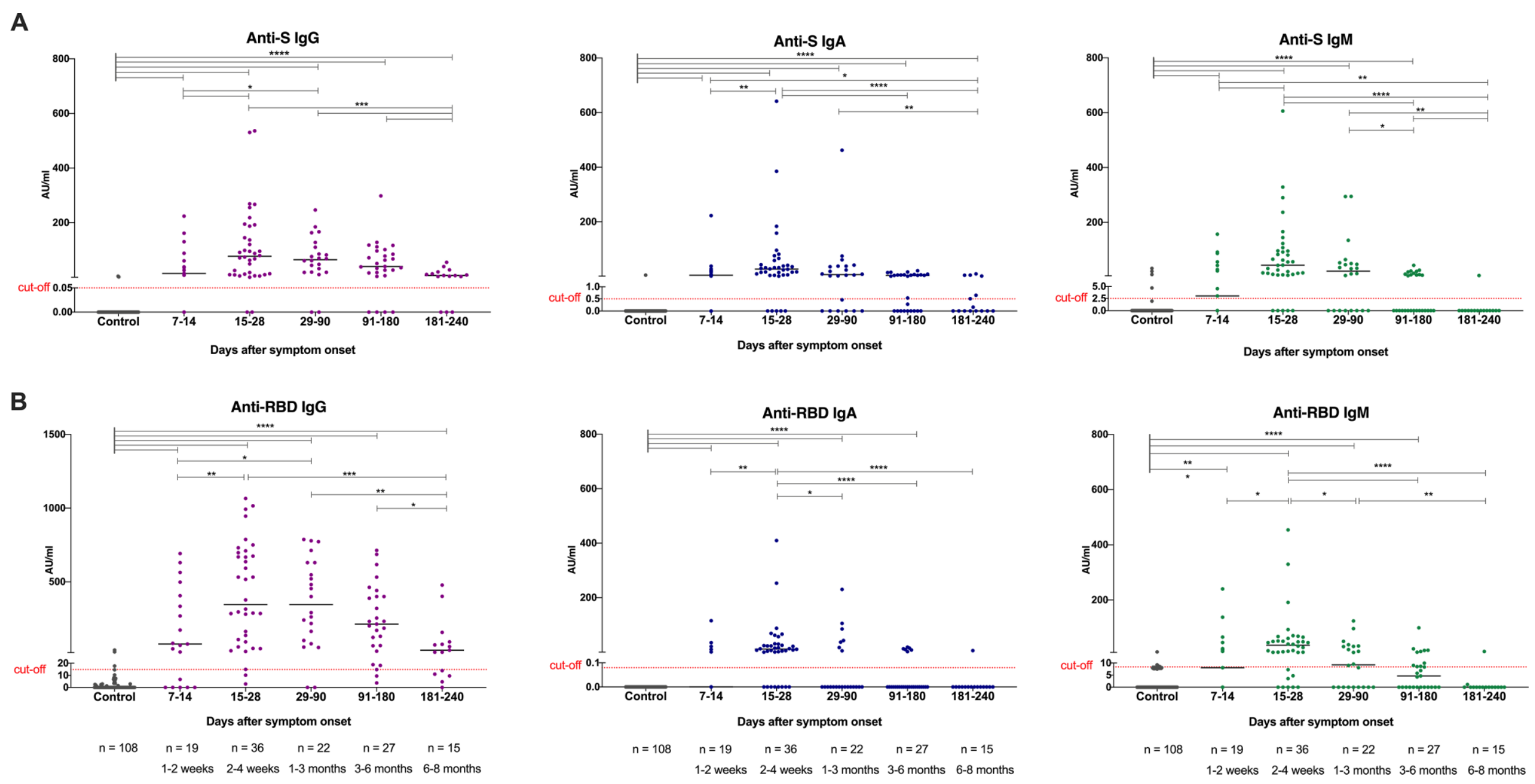
Figure 4

Anti-S IgG

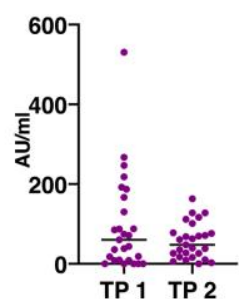

Anti-S IgA

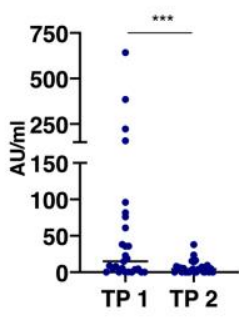

Anti-S IgM

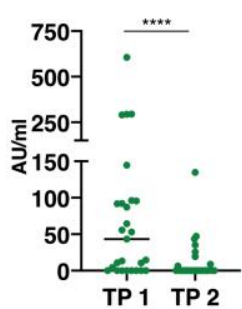

Anti-S IgG

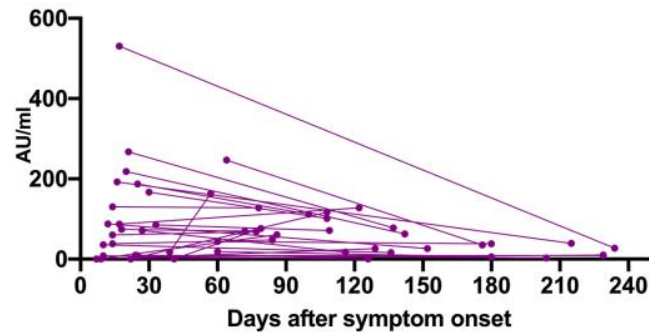

Anti-S IgA

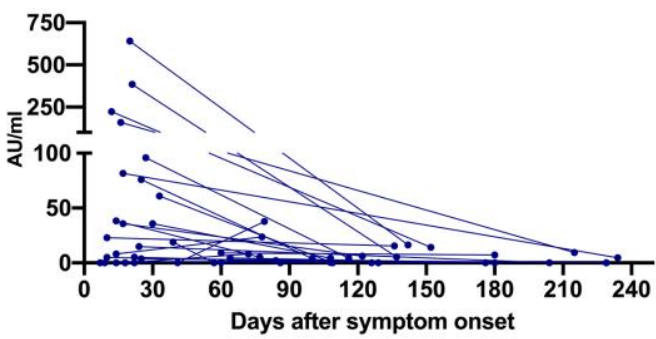

Anti-S IgM

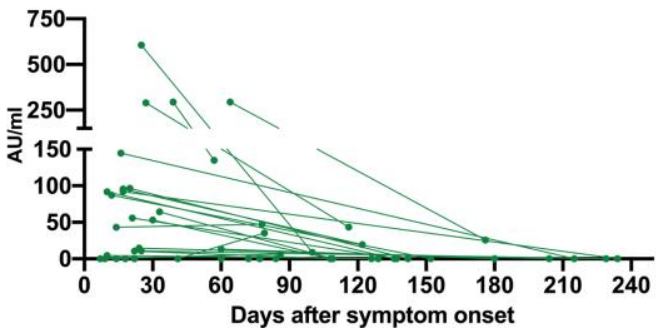

Anti-RBD IgG

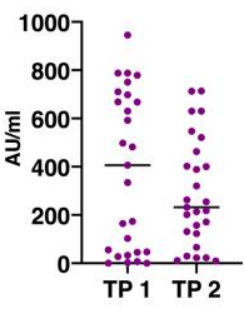

Anti-RBD IgA

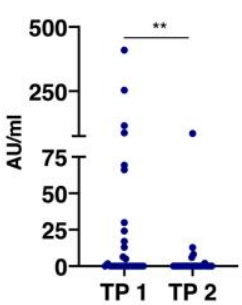

Anti-RBD IgM

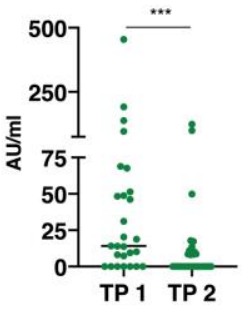

Anti-RBD IgG

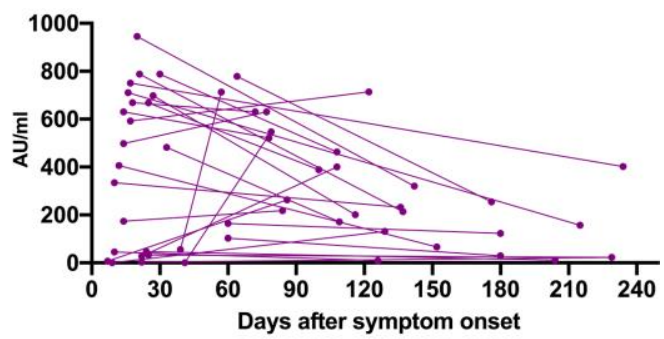

Anti-RBD IgA

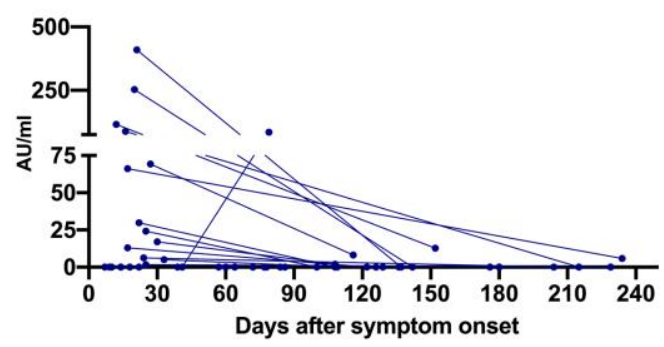

Anti-RBD IgM

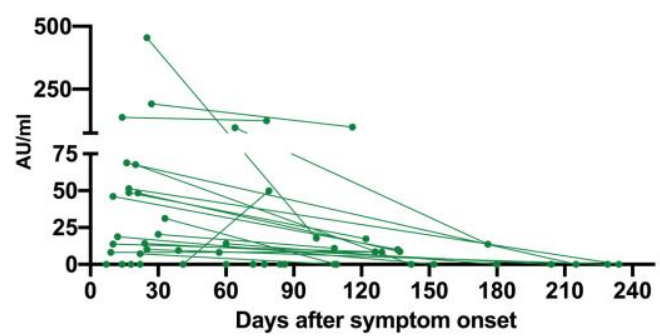


Figure 5
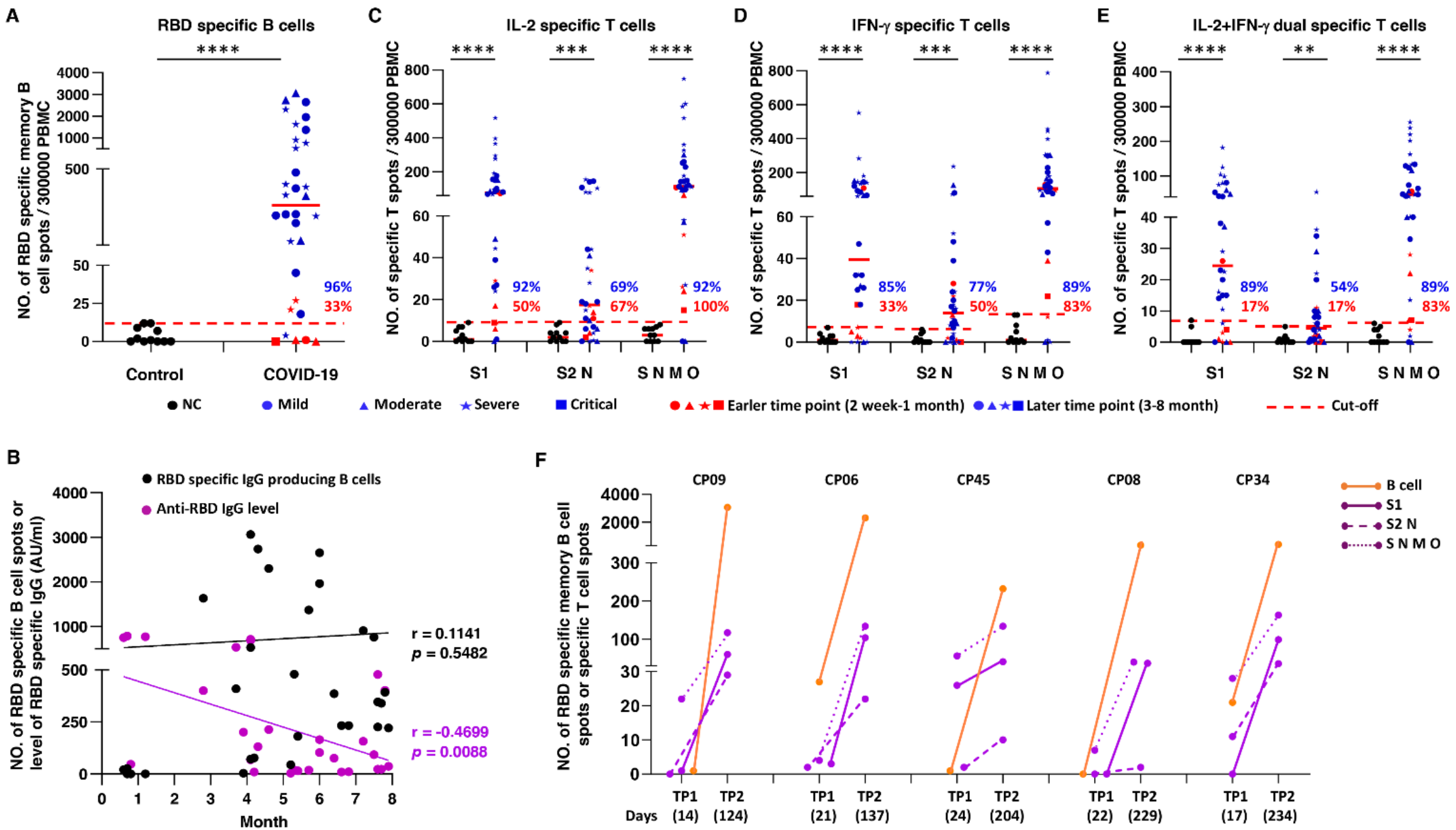